\title{
Serum Level of Thyroid Hormones in Patients with Chronic Hepatitis C Virus Infection
}

\author{
Mohamed Abdel-Fattah El-Feki, Nilly Helmy Abdalla, Mohamed Ibrahim Atta, \\ Ahmed Amin Ibrahim \\ Internal Medicine Department, Faculty of Medicine, Beni Suef University, Beni Suef, Egypt \\ Email: drmfeki@hotmail.com, nillyhelmy@yahoo.com,mohamedatta1975@yahoo.com, \\ drahmedamin201096@yahoo.com
}

Received 7 February 2016; accepted 20 March 2016; published 23 March 2016

Copyright (C) 2016 by authors and Scientific Research Publishing Inc.

This work is licensed under the Creative Commons Attribution International License (CC BY).

http://creativecommons.org/licenses/by/4.0/

(c) (i) Open Access

\begin{abstract}
Objective: There are clinical and laboratory associations between thyroid and liver diseases. Hepatitis C virus (HCV) is known to be responsible for both hepatic and extrahepatic diseases. The most frequent and clinically important endocrine extrahepatic diseases are thyroid disorders and type 2 diabetes mellitus. We aim to study the relationship between the serum level of thyroid hormones (THs) and the severity of liver disease in patients with chronic hepatitis C virus (CHC) infection. Methods: 60 patients with CHC infection were selected for the study. They were divided into two groups: with or without liver cirrhosis. Those with liver cirrhosis were further subdivided according to the Child-Turcotte-Pugh scoring system. Serum levels of free T3 (FT3), free T4 (FT4) and TSH were measured to all patients. Results: There was decrease in the FT3 and FT4 levels and increase in the TSH levels in patients with CHC with cirrhosis when compared to patients with CHC without cirrhosis. Conclusion: Thyroid profile abnormalities were seen in cirrhotic HCV patients when compared to non-cirrhotic patients. The abnormalities in the serum level of THs (decreased FT3, FT4, and increased TSH) are strongly associated with the severity of liver damage and advancing of the child score.
\end{abstract}

\section{Keywords}

Hepatitis C Virus, Thyroid Hormones, Liver Cirrhosis

\section{Introduction}

Egypt has the highest prevalence of HCV infection in the world, estimated nationally at $14.7 \%$ [1]. Approximately $70 \%-80 \%$ of $\mathrm{HCV}$-infected patients fail to clear the virus and develop $\mathrm{CHC}$ infection, which is a risk 
factor for liver diseases such as fibrosis, cirrhosis, and hepatocellular carcinoma [2] [3]. In addition to its hepatic manifestations, $\mathrm{CHC}$ infection is associated with extrahepatic manifestations occurring in multiple organ systems, including the hematologic, renal, dermatologic, endocrine and rheumatologic systems. Among the endocrine disorders, thyroid disorders are the most common and the thyroid is one of the principal target organs for extrahepatic manifestations in HCV-infected patients [4]. The liver plays an important role in the metabolism of THS, as it is the most important organ in the peripheral conversion of tetraiodothyronine (T4) to triiodothyronine (T3) by type 1 deiodinase [2] [5]. Moreover, the liver is involved in thyroid hormone conjugation and excretion, as well as the synthesis of thyroid binding globulin [2] [6].

T4 and T3 regulate the basal metabolic rate (BMR) of all cells, including hepatocytes, and thereby modulate hepatic function. The liver metabolizes the THs and regulates their systemic endocrine effects. Thyroid diseases may perturb liver function; liver disease modulates thyroid hormone metabolism; and a variety of systemic diseases affect both organs [7]. There are clinical and laboratory associations between thyroid and liver diseases. Patients with chronic liver disease may have thyroiditis, hyperthyroidism, or hypothyroidism. Patients with subacute thyroiditis or hyperthyroidism may have abnormalities in liver function tests, which return to normal as the thyroid condition improves [7]. HCV is known to be responsible for both hepatic and extrahepatic diseases. The most frequent and clinically important endocrine extrahepatic diseases are thyroid disorders and type 2 diabetes mellitus [8].

\section{Patients and Methods}

\subsection{Patients}

60 patients with chronic hepatitis $\mathrm{C}$ (CHC) infection were selected for the study; they were followed up at outpatient clinics at Beni Suef University Hospital and Beni Suef General Hospital. We excluded the following patient from study; patients who tested positive for serum HBs Ag, patients suffering from previously known thyroid disease, patients who were currently under treatment or were previously treated with thyroid stimulating/ inhibiting agents, patients who were currently under treatment or were previously treated with interferon therapy, patients on medications that could affect the study outcome including Carbamazepine, Phenobarbitone, Phenytoin, Salicylates and Nonsteroidal anti-inflammatory drugs (NSAIDs) and patients with a history of alcohol consumption in the previous six months. The patients who met these criteria were divided into two groups: Group I: 15 patients with CHC infection without liver cirrhosis and Group II: 45 patients with CHC infection with liver cirrhosis. Group II was classified according to the Child-Turcotte-Pugh scoring system into Child A (15 patients), Child B (15 patients), and Child C (15 patients).

\subsection{Methods}

All the patients in the study were subjected to the following; informed consent; history taking such as history of $\mathrm{HCV}$ infection or thyroid disorder, treatment or history of IFN therapy, or history of other diseases or other special habits; clinical assessment including symptoms and signs of hepatic decompensation (e.g., encephalopathy, ascites and jaundice or manifestations of thyroid disorder or other manifestations in other systems). Investigations including abdominal ultrasound, laboratory tests were performed and included: liver function tests (aspartate aminotransferase or AST, ALT, serum albumin, serum bilirubin, prothrombin time and concentration, INR), serum anti-HCV antibody, HCV RNA PCR, serum HBs-Ag, complete blood count, thyroid functions (FT3, FT4, and TSH), using the enzyme linked immunosorbent assay (ELISA) kit provided by ACCU-Bind ELISA kit, USA.

\subsection{Statistical Analysis}

All statistical calculations were done by computer using the Microsoft Excel 2010 program and the Statistical Package for the Social Sciences (SPSS) software version 17 for Microsoft Windows. The following tests were used; descriptive analysis of the results in the form of percentage distribution for qualitative data and calculation of minimum, maximum, mean, and standard deviation for quantitative data, cross tabulation test: for comparison between percentage values, student's t-test: for comparison between means of two groups, the Chi-square test: used to test the statistical significance of differences in a classification system (one-way classification) or the relationship between two classification systems (two-way classification), Fisher's exact test: used to calculate an 
exact P-value for a $2 \times 2$ frequency table with a small number of expected frequencies, for which the Chisquare test is not appropriate. Mann-Whitney $U$ test for two independent samples: a nonparametric test for a betweensubjects design using two levels of an independent variable and scores that are measured at least at the ordinal level.

\section{Results}

Regarding age and gender, there were no statistically significant differences between the non-cirrhotic and the cirrhotic groups, as shown in Table 1.

Regarding the thyroid profile, there was a statistical highly significant difference in TSH and a statistically significant difference in FT3 and FT4 between the groups, as shown in Figure 1.

Regarding age, there was a statistically significant difference in age between patients in Child A, B, and C; between patients in Child A and C; and between patients in Child B and C. However, there was no statistically significant difference between patients in Child A and B, Table 2 and Figure 2, Figure 3. The following abbreviations are used in this section:

- P: Comparison between Child A, B, and C;

- $\mathrm{P}_{1}$ : Comparison between Child A and Child B;

- $\mathrm{P}_{2}$ : Comparison between Child A and Child C;

- $\mathrm{P}_{3}$ : Comparison between Child B and Child C.

Table 1. Comparison of demographic data between non-cirrhotic and cirrhotic groups.

\begin{tabular}{|c|c|c|c|c|c|c|}
\hline Variables & \multicolumn{2}{|c|}{ Non-cirrhotic group } & \multicolumn{2}{|c|}{ Cirrhotic group } & & \\
\hline \multicolumn{7}{|l|}{ Age (years) } \\
\hline $\mathrm{X} \pm \mathrm{SD}$ & \multicolumn{2}{|c|}{$47.5 \pm 8.8$} & \multicolumn{2}{|c|}{$51.8 \pm 8.2$} & $\mathrm{t}$ & $\mathrm{P}$ \\
\hline Range & \multicolumn{2}{|c|}{$27-60$} & \multicolumn{2}{|c|}{$33-65$} & 2.1 & 0.089 \\
\hline Gender & $\mathrm{N}$ & $\%$ & $\mathrm{~N}$ & $\%$ & $\mathrm{X}^{2}$ & $\mathrm{P}$ \\
\hline Male & 9 & 60 & 30 & 66.7 & 0.22 & 0.639 \\
\hline Female & 6 & 40 & 15 & 33.3 & & \\
\hline
\end{tabular}

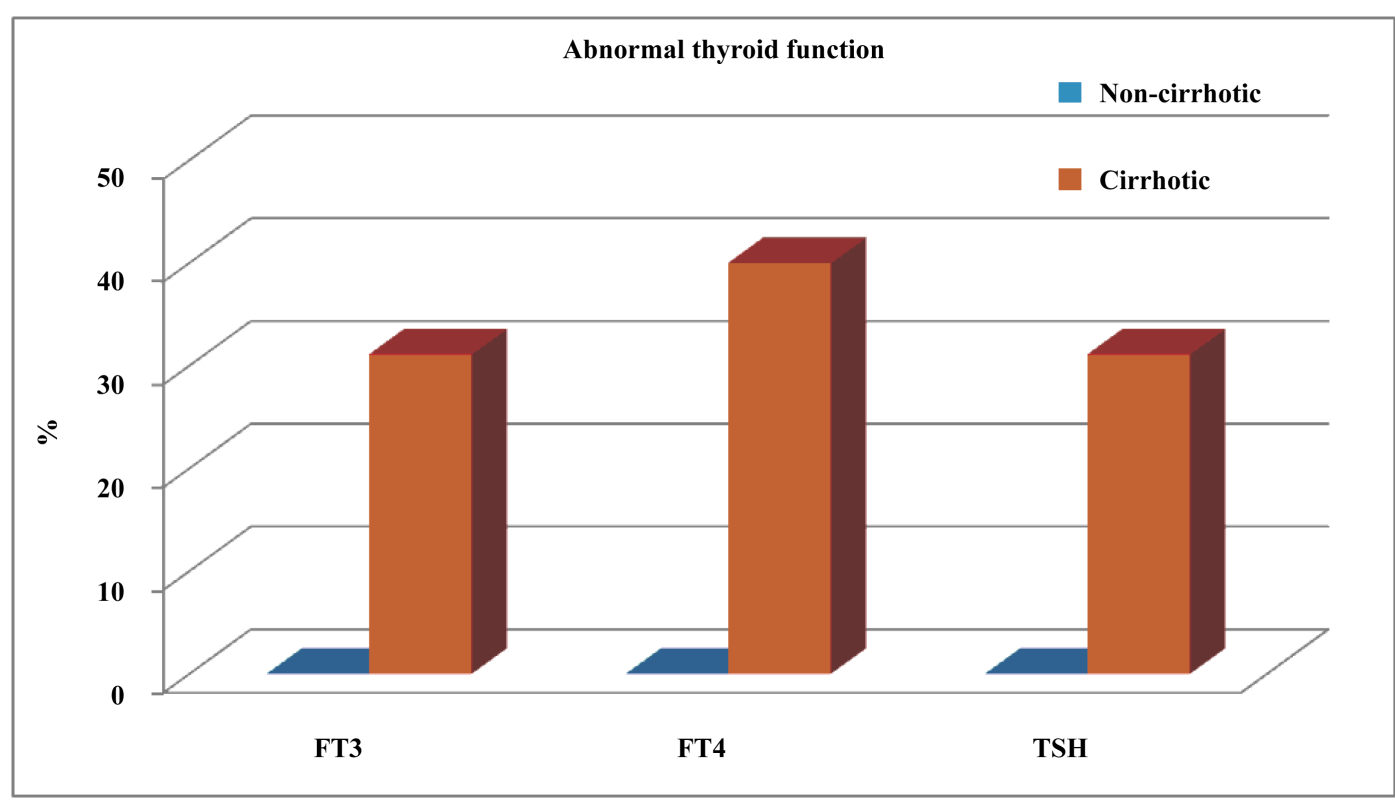

Figure 1. Distribution of abnormal thyroid functions between non-cirrhotic and cirrhotic group. 
Table 2. Comparison of demographic data within the cirrhotic group.

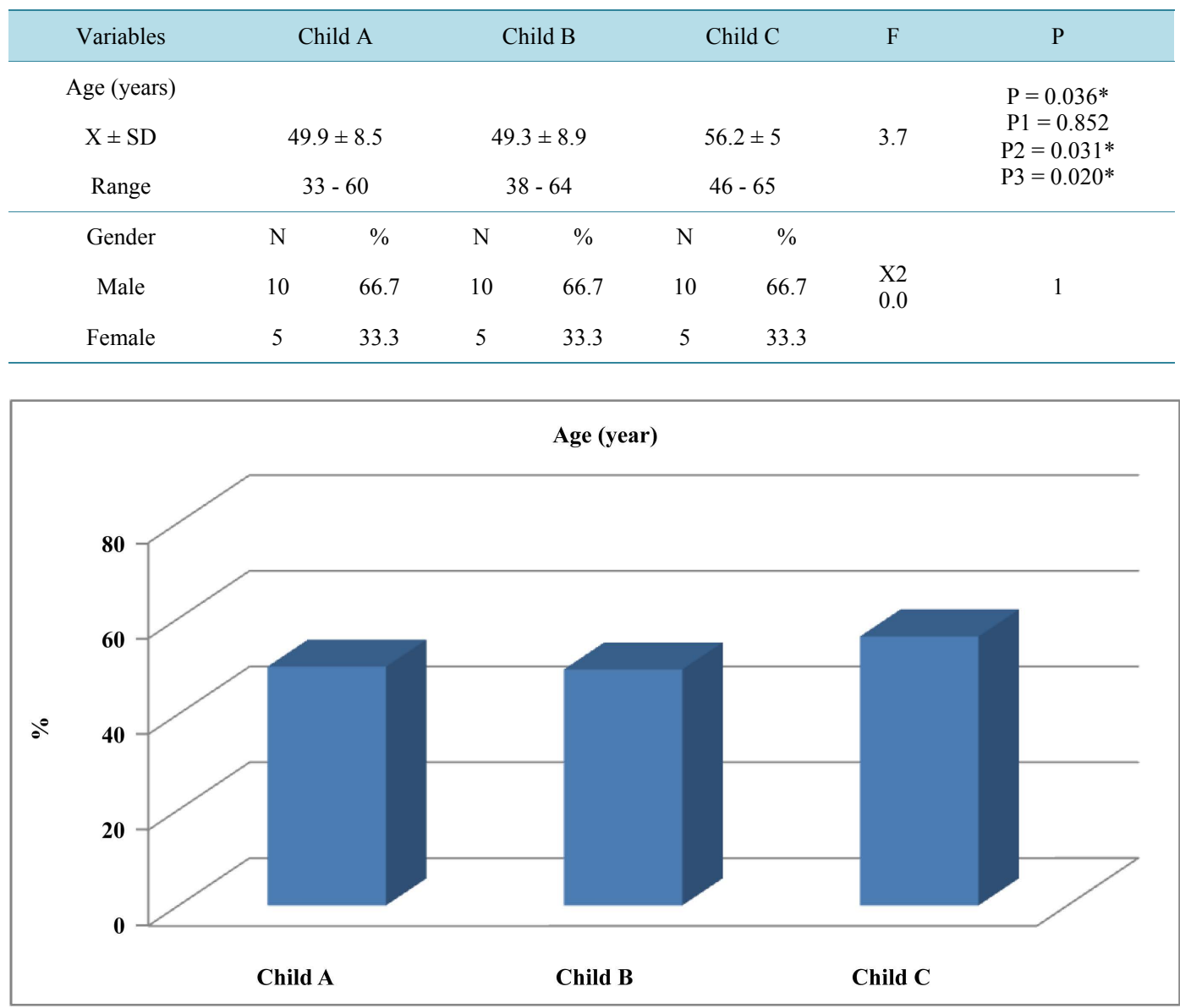

Figure 2. Comparison of age within the cirrhotic group.

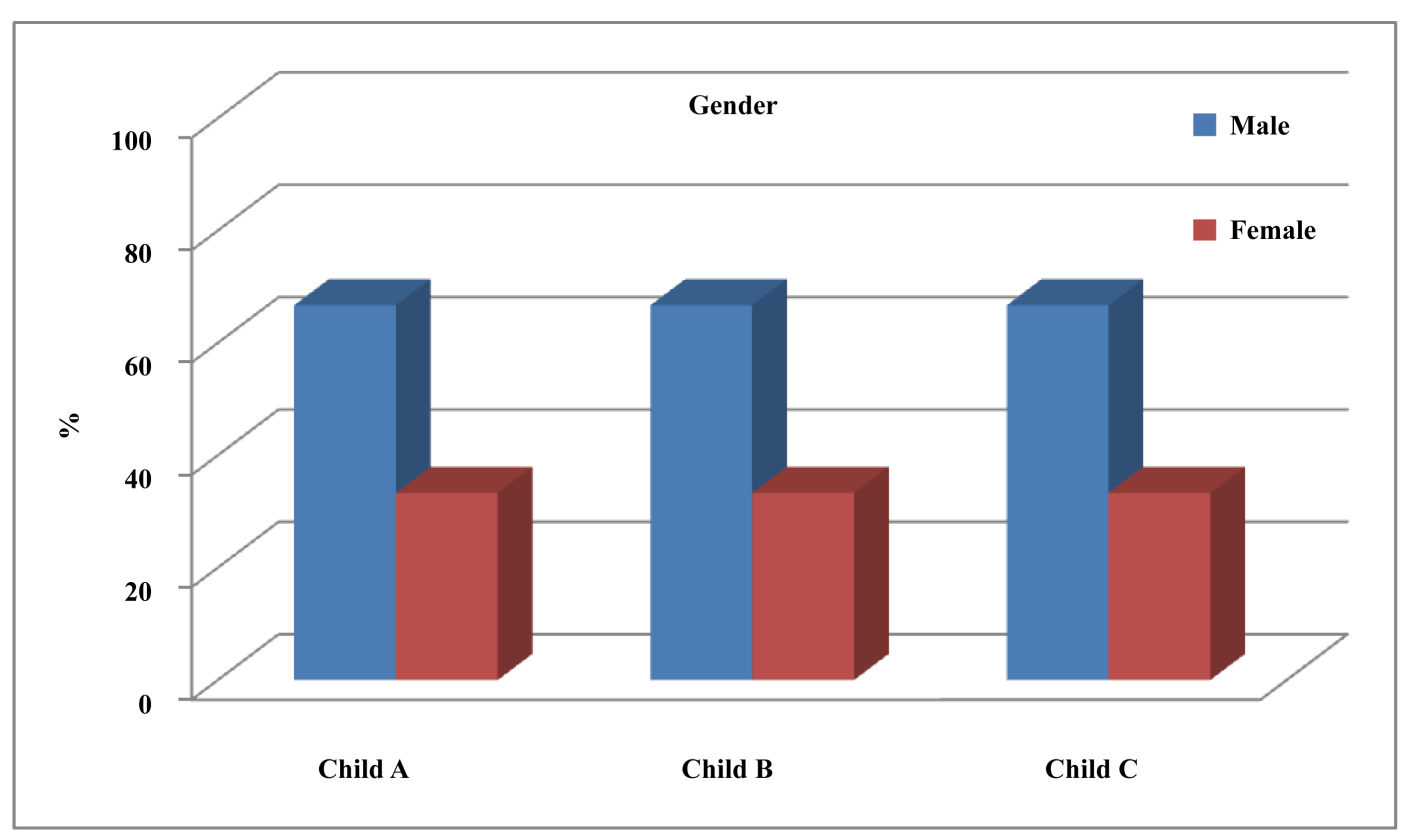

Figure 3. Comparison of gender within the cirrhotic group. 
Regarding the thyroid profile FT3, FT4, and TSH there was a statistically significant difference between the cirrhotic groups, except between Child A and Child B as shown in Table 3, Figure 4.

Regarding the correlation between the thyroid profile and other variables, FT3 showed a statistically significant positive correlation with platelets, albumin, and $\mathrm{PC} \%$ and an inverse correlation with ALT and T. bilirubin. FT4 showed a significant positive correlation with $\mathrm{PC} \%$ and an inverse correlation with $\mathrm{T}$. bilirubin. TSH showed a significant positive correlation with ALT and T. bilirubin, and an inverse correlation with platelets, albumin, and PC\%, as shown in Table 4 and Figures 5-7.

Table 3. Comparison of thyroid profile within the cirrhotic group.

\begin{tabular}{|c|c|c|c|c|c|}
\hline & Child A & Child B & Child C & $\mathbf{F}$ & $\mathbf{P}$ \\
\hline FT3 & & & & \multirow{3}{*}{6.3} & \multirow{3}{*}{$\begin{array}{c}\mathrm{P}=0.004^{* *} \\
\mathrm{P}_{1}=0.078 \\
\mathrm{P}_{2}=0.001 * * \\
\mathrm{P}_{3}=0.048^{*}\end{array}$} \\
\hline $\mathrm{X} \pm \mathrm{SD}$ & $2.5 \pm 0.6$ & $1.9 \pm 1.0$ & $1.4 \pm 1.0$ & & \\
\hline Range & $1.4-3.6$ & $0.1-3.3$ & $0.2-3.7$ & & \\
\hline FT4 & & & & \multirow{3}{*}{4.1} & \multirow{3}{*}{$\begin{array}{c}\mathrm{P}=0.023^{*} \\
\mathrm{P}_{1}=0.420 \\
\mathrm{P}_{2}=0.046^{*} \\
\mathrm{P}_{3}=0.008^{*} *\end{array}$} \\
\hline $\mathrm{X} \pm \mathrm{SD}$ & $1.1 \pm 0.4$ & $1.3 \pm 0.9$ & $0.7 \pm 0.4$ & & \\
\hline Range & $0.6-2.1$ & $0.3-2.6$ & $0.2-1.9$ & & \\
\hline TSH & & & & \multirow{4}{*}{17.7} & \multirow{4}{*}{$\begin{array}{c}\mathrm{P}=<0.001 * * \\
\mathrm{P}_{1}=0.529 \\
\mathrm{P}_{2}=0.001 * * \\
\mathrm{P}_{3}=0.001 * *\end{array}$} \\
\hline $\mathrm{X} \pm \mathrm{SD}$ & $1.5 \pm 1.2$ & $3.3 \pm 3.1$ & $18.1 \pm 14.3$ & & \\
\hline Range & $0.3-4.3$ & $0.3-8.6$ & $0.9-45.7$ & & \\
\hline Median & 0.6 & 1.9 & 21.5 & & \\
\hline
\end{tabular}

Table 4. Correlation between thyroid profile and other variables among all cases.

\begin{tabular}{|c|c|c|c|c|c|c|}
\hline & \multicolumn{2}{|c|}{ FT3 } & \multicolumn{2}{|c|}{ FT4 } & \multicolumn{2}{|c|}{ TSH } \\
\hline & $\mathbf{r}$ & P-value & $\mathbf{r}$ & P-value & $\mathbf{r}$ & P-value \\
\hline TLC & 0.01 & 0.909 & 0.01 & 0.965 & 0.1 & 0.489 \\
\hline Platelets & 0.4 & $0.005 * *$ & 0.2 & 0.291 & -0.5 & $0.001 * *$ \\
\hline $\mathbf{A L T}$ & -0.3 & $0.037 *$ & -0.2 & 0.211 & 0.5 & $0.001 * *$ \\
\hline Albumin & 0.5 & $0.001 * *$ & 0.2 & 0.133 & -0.6 & $0.001 * *$ \\
\hline T. bilirubin & -0.4 & $0.006^{* *}$ & -0.4 & $0.013 *$ & 0.7 & $0.001 * *$ \\
\hline PC\% & 0.5 & $0.001 * *$ & 0.4 & $0.004 * *$ & -0.7 & $0.001 * *$ \\
\hline
\end{tabular}

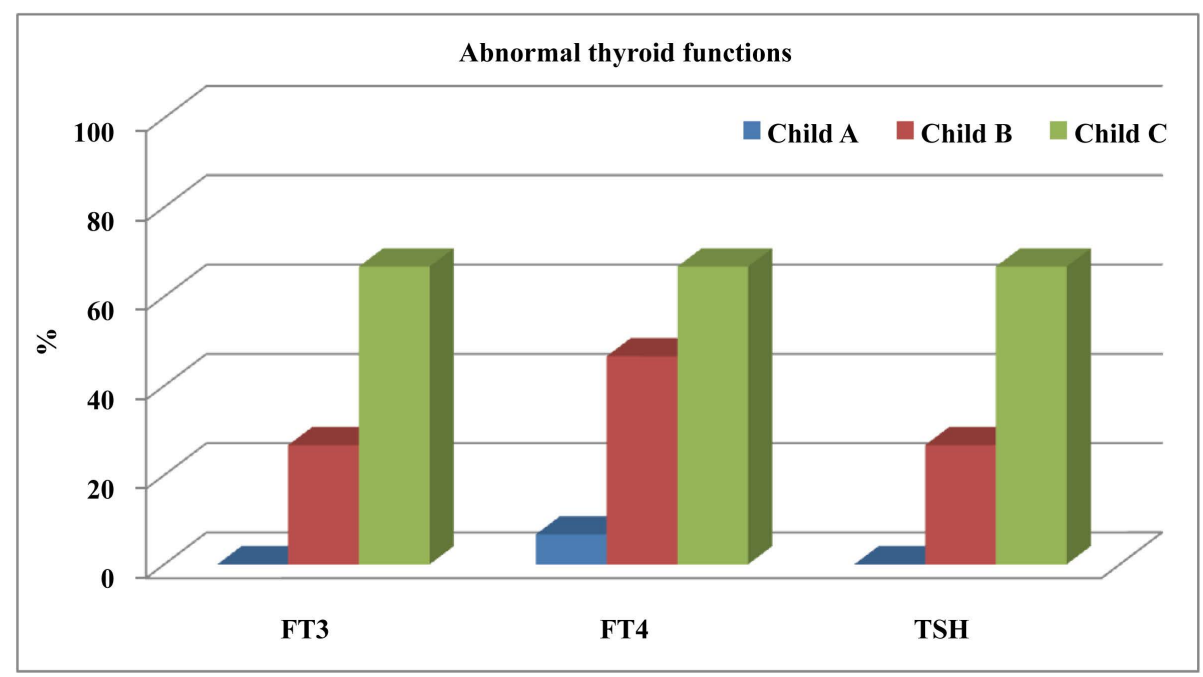

Figure 4. Distribution of abnormal thyroid functions within the cirrhotic group. 


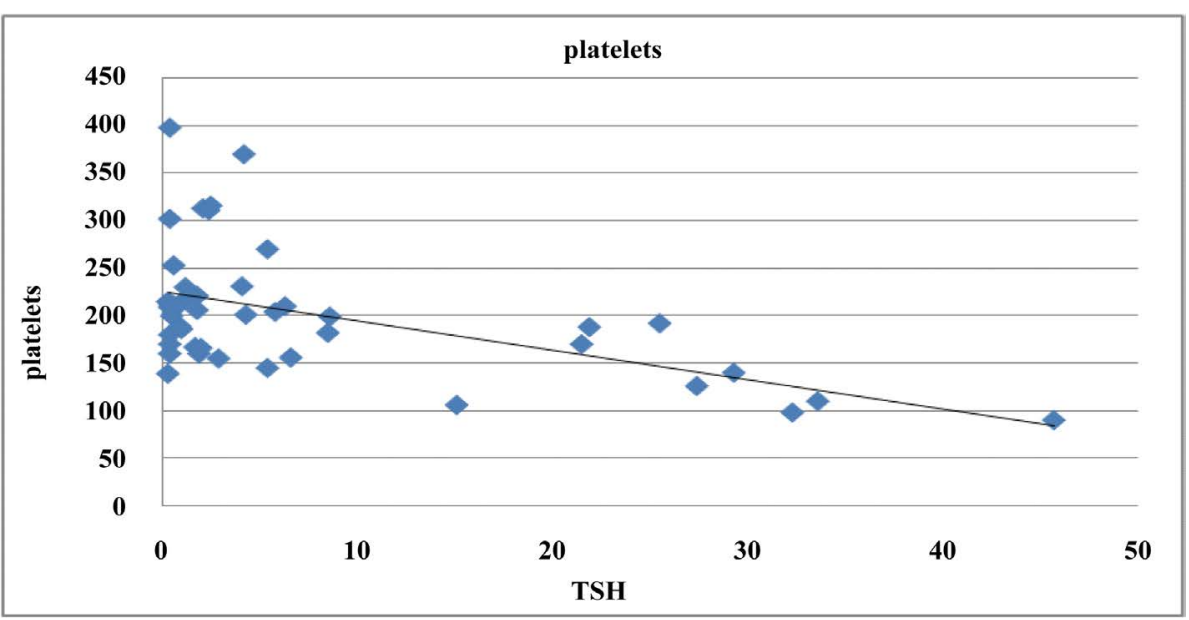

Figure 5. Correlation between TSH and platelets.

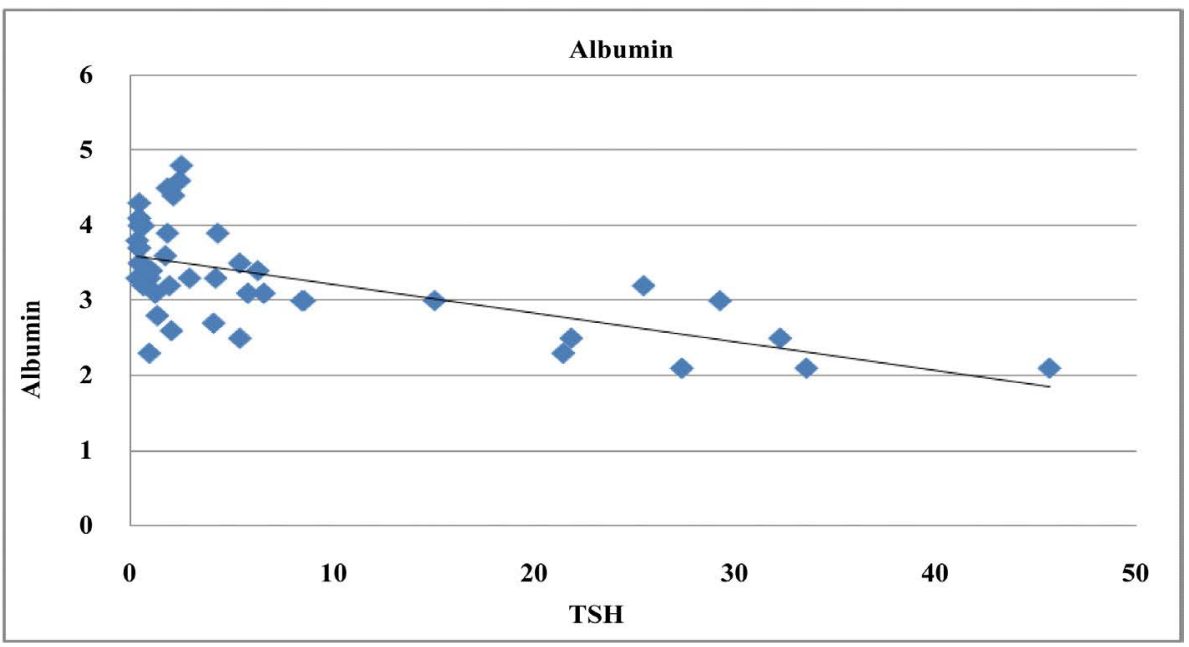

Figure 6. Correlation between TSH and albumin.

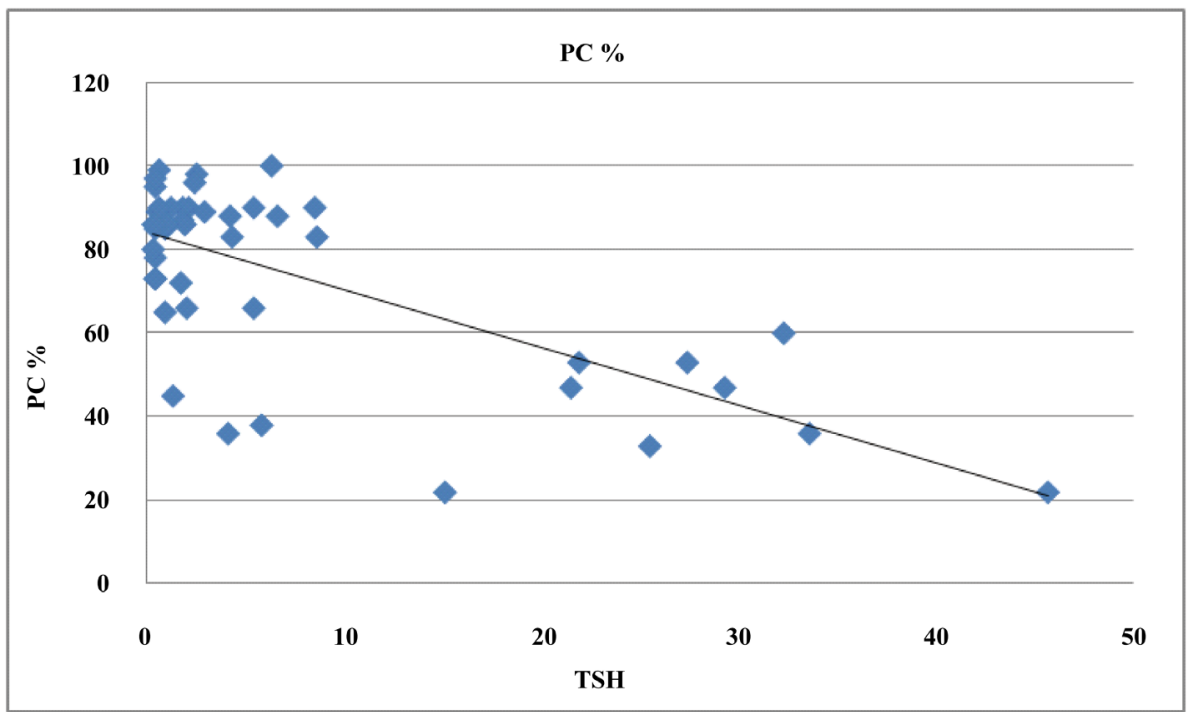

Figure 7. Correlation between TSH and PC\%. 


\section{Discussion}

The liver plays an important role in the metabolism of THs, as it is the most important organ in the peripheral conversion of T4 to T3 by D1 [2]. Moreover, it is involved in conjugation and circulation of THs by synthesis of thyroid binding proteins [6]. There is evidence of an association between chronic liver diseases and changes in the thyroid gland. It is demonstrated that levels of THs and their binding proteins are altered in patients with hepatic disorders, especially cirrhosis. The most frequent change in plasma levels of THs is a decrease in total T3 and FT3 concentrations, which is reported to be associated with the severity of hepatic dysfunction [9].

$\mathrm{T} 4$ and $\mathrm{T} 3$ regulate the BMR of all cells, including hepatocytes, and thereby modulate hepatic function. The liver metabolizes the THs and regulates their systemic endocrine effects. TD may perturb liver function; liver disease modulates thyroid hormone metabolism; and a variety of systemic diseases affect both organs [7]. The low total and FT3 levels may be regarded as an adaptive hypothyroid state that serves to reduce the basal BMR within hepatocytes and preserve liver function and total body protein stores [7].

In our study the total number of patients was 60 patients; the patients were divided into two groups: Group I (15 patients) had CHC infection without liver cirrhosis (non-cirrhotic group); Group II (45 patients) had CHC infection with liver cirrhosis (cirrhotic group). Group II (cirrhotic group) was further subdivided according to the CTP scoring system into Child A (15 patients), Child B (15 patients) and Child C (15 patients).

In the statistical analysis, Group I (non-cirrhotic group) was compared with Group II (cirrhotic group); also patients classified as Child A, B and C was compared within the cirrhotic group.

In the present study, when the age and gender of patients in Group I and Group II were compared, there were no statistically significant differences between the two groups.

The results of the present study showed a statistically significant decrease in FT3 level in patients with CHC with cirrhosis (Group II), when compared to patients with CHC without cirrhosis (Group I) $(\mathrm{P}=0.039)$.

To date, studies and reports unanimously agree that patients with liver cirrhosis have low serum total T3 and FT3.

The present study is in agreement with [10], who found that the dysregulation and dysfunction of THs were expected in patients with liver cirrhosis. Low total and FT3 are the most common pattern of thyroid hormone abnormalities. This is probably due to reduced D1 activity and subsequent impaired hepatic conversion of T4 to T3. It is also in agreement with [9], who found that levels of THs and their binding proteins were altered in patients with hepatic disorders, especially cirrhosis. The most frequent changes in plasma levels of THs are decreases in total T3 and FT3 concentrations. In addition, it is in agreement with [11], who found that FT3 levels were significantly lower in patients with CHC. Both hypothyroidism and thyroid autoimmunity are more common in patients with CHC, even in the absence of cirrhosis. It also agrees with [7], who suggested that low total and FT3 levels might be regarded as an adaptive hypothyroid state that serves to reduce the BMR within hepatocytes and preserve liver function and total body protein stores.

The results of the present study showed a statistically significant decrease in FT4 level in patients with CHC with cirrhosis (Group II) when compared to patients with CHC without cirrhosis (Group I) (P = 0.019).

This is in agreement with [11], who found that FT4 levels were significantly lower in patients with CHC even in the absence of cirrhosis. This finding is also in agreement with [12], who found low levels of T4 and T3 along with extremely high rT3 in patients with advanced liver cirrhosis; they also observed high mortality in these patients and explained this by altered T4 metabolism with lowering of T4 and T3 along with a rise in rT3. They suggested that these changes might be dependent on the degree of hepatocellular damage and reverse on improvement in liver function; and they considered T4, T3, and rT3 levels to be useful prognostic indices.

On the other hand, this disagrees with [7], who concluded that the majority of patients with liver disease were clinically euthyroid and this could be confirmed by a normal TSH and a normal FT4.

The results of the present study showed a highly significant statistical increase in the TSH level in patients with $\mathrm{CHC}$ with cirrhosis (Group II) when compared to patients with $\mathrm{CHC}$ without cirrhosis (Group I) (P = 0.001 ).

This is in agreement with [11] who suggested that abnormality in TSH secretion was due to the existence of hypothalamic-pituitary dysfunction in advanced liver disease. Several lines of evidence suggest a reduced dopaminergic tone as a consequence of the accumulation of false neurotransmitters, which might be responsible for raised basal TSH concentrations, as dopamine has been shown to exert an inhibitory effect in the regulation of TSH secretion. It is also in agreement with [13], who found that the serum TSH level was elevated in decom- 
pensated cirrhotic HCV patients when compared to both chronic and compensated cirrhotic HCV patients. Moreover, this agrees with ([11] et al. 2004), who found that the level of TSH was significantly higher in patients with CHC. Finally, it is in agreement with [9], who found a positive correlation between TSH levels and CTP scores in patients with $\mathrm{CHC}$ with cirrhosis.

However, it disagrees with [6], who found no significant difference in TSH in cirrhotic patients. The present study found a statistically significant difference in age between Child $\mathrm{A}, \mathrm{B}$, and $\mathrm{C}(\mathrm{P}=0.036)$; Child $\mathrm{A}$ and $\mathrm{C}$ ( $\mathrm{P}$ $=0.031)$; Child $\mathrm{B}$ and $\mathrm{C}(\mathrm{P}=0.020)$. However, there was no statistically significant difference between Child A and $\mathrm{B}(\mathrm{P}=0.852)$. There was also no statistically significant difference in gender among the groups $(\mathrm{P}=1)$.

The results of the present study showed that there was a statistically significant difference in the FT3 level between the groups (except between Child A and B) as follows: Child A, B, and C $(\mathrm{P}=0.004)$; Child A and B $(\mathrm{P}=0.078)$; Child $\mathrm{A}$ and $\mathrm{C}(\mathrm{P}=0.001)$; and Child $\mathrm{B}$ and $\mathrm{C}(\mathrm{P}=0.048)$. The lack of a statistically significant difference between Child A and B was probably due to the fact that the patients were in the early stages of Child A and Child B.

On comparing the mean serum levels of FT3 in Child A, B, and C, the lowest levels were among the Child C group $(1.4 \pm 1.0)$, followed by the Child B group $(1.9 \pm 1.0)$, while the Child A group was within the normal range $(2.5 \pm 0.6)$. This is in agreement with [5], who found that serum total T3 was lower in all forms of liver disease when compared with controls. However, FT3 was significantly lower in patients with chronic liver disease, especially liver cirrhosis (Child B or C). This was due to decreased hepatic uptake and D1 activity, which converted T4 to T3. The present results were also in agreement with [14], who found that alterations in thyroid hormone regulation and metabolism were frequently observed in patients with liver cirrhosis with a decreased serum T3 concentration. In severe cases, low T4 concentrations are also observed. It is also in agreement with [15], who found that the most common thyroid hormone profile in patients with cirrhosis was low total T3 and FT3 along with an elevated rT3, similar to changes in those suffering from the sick euthyroid syndrome, reflecting a reduced D1 activity, resulting in reduced conversion of T4 to T3. In addition, the results were in agreement with [16], who found a significant decrease in FT3 and a significant increase in FT4 in both a cirrhotic group of patients and a CHC group.

On comparing the mean serum level of FT4 between Child A, B, and C, the lowest levels found were in the Child C group $(0.7 \pm 0.4)$, followed by the Child B group $(1.3 \pm 0.9)$, while the Child A group was within the normal range $(1.1 \pm 0.4)$. However, in the Child B group $(1.3 \pm 0.9)$, FT4 was low in four patients and high in three patients. This could be explained by [17], who suggested that HCV may contribute to the occurrence of thyroid autoantibodies and in some cases initiate autoimmune thyroid disorders. In addition, thyroid autoantibodies may induce a hypothyroid or hyperthyroid state.

The present results are in agreement with [18], who found that in cases of prolonged illness, hypothalamicpituitary suppression usually occurred. This leads to decreased secretion of TSH, decreased T4 production by the thyroid gland, and a subsequent decline of FT4 levels. This is also in agreement with [14], who found that alterations in thyroid hormone regulation and metabolism were frequently observed in patients with liver cirrhosis with decreased serum T3 concentration. In severe cases, low T4 concentrations are also observed. Finally, it is in agreement with [6], who found that patients with decompensated liver disease had exceedingly low serum FT3 and T4 levels.

The present results disagree with [19], who found FT4 and TSH levels were usually normal in patients with chronic liver disease. It conflicts too with [11], who found a significant decrease in FT3 and significant increase in FT4 in both a cirrhotic group and a CHC group. These conflicting results might be explained by differences in geographic distribution, genetic background of the population, and environmental factors, such as iodine intake or other infectious agents.

When comparing the mean serum levels of TSH in Child A, B, and C, it was found to be the highest in the Child C group $(18.1 \pm 14.3)$, followed by the Child B group $(3.3 \pm 3.1)$, while the Child A group was within the normal range $(1.5 \pm 1.2)$.

This is in agreement with [13], who found that the serum TSH level was elevated in decompensated cirrhotic HCV patients when compared to both chronic and compensated cirrhotic HCV patients. It is also in agreement with [11], who found that the level of TSH was significantly higher in patients with CHC. In addition, it is in agreement with [9], who found a positive correlation between TSH levels and CTP scores in patients with CHC with cirrhosis.

However, it disagrees with [5], who found lower TSH levels in patients with chronic liver disease compared 
to controls. They suggested this was because there was an increased level of inflammatory cytokines that negatively affected the hypothalamic-thyroid axis in chronic liver disease. It also disagrees with [6], who found that TSH levels showed no significant difference in cirrhotic patients. These conflicting results might be due to differences in geographic distribution, genetic background of the population, and environmental factors, such as iodine intake or other infectious agents.

\section{References}

[1] Mohamoud, Y.A., Mumtaz, G.R., Riome, S., Miller, D. and Abu-Raddad, L. (2013) The Epidemiology of Hepatitis C Virus in Egypt: A Systematic Review and Data Synthesis. BMC Infectious Diseases, 13, 288. http://dx.doi.org/10.1186/1471-2334-13-288

[2] Sorvillo, F., et al. (2003) Increased Serum Reverse Triiodothyronine Levels at Diagnosis of Hepatocellular Carcinoma in Patients with Compensated HCV-Related Liver Cirrhosis. Clinical Endocrinology, 58, 207-212. http://dx.doi.org/10.1046/j.1365-2265.2003.01697.x

[3] Lara, J., López-Labrador, F.X., González-Candelas, F., Berenguer, M. and Khudyakov, Y.E. (2014) Computational Models of Liver Fibrosis Progression for Hepatitis C Virus Chronic Infection. BMC Bioinformatics, 15, S5. http://dx.doi.org/10.1186/1471-2105-15-S8-S5

[4] Jadali, Z. (2013) Autoimmune Thyroid Disorders in Hepatitis C Virus infection: Effect of Interferon Therapy. Indian Journal of Endocrinology and Metabolism, 17, 69-75. http://dx.doi.org/10.4103/2230-8210.107856

[5] Kharb, S., Garg, M.K., Puri, P., Brar, K.S., Pandit, A. and Srivastava, S. (2015) Assessment of Thyroid and Gonadal Function in Liver Diseases. Indian Journal of Endocrinology and Metabolism, 19, 89-94. http://dx.doi.org/10.4103/2230-8210.131761

[6] Kayacetin, E., Kisakol, G. and Kaya, A. (2003) Low Serum Total Thyroxine and Free Triiodothyronine in Patients with Hepatic Encephalopathy due to Non-Alcoholic Cirrhosis. Swiss Medical Weekly, 133, 210-213.

[7] Malik, R. and Hodgson, H. (2002) The Relationship between the Thyroid Gland and the Liver. QJM, 95, 559-569. http://dx.doi.org/10.1093/ajmed/95.9.559

[8] Fallahi, P., et al. (2014) Targeting Chemokine (C-X-C Motif) Receptor 3 in Thyroid Autoimmunity. Recent Patents on Endocrine, Metabolic \& Immune Drug Discovery, 8, 95-101. http://dx.doi.org/10.2174/1872214808666140623114315

[9] Mansour-Ghanaei, F., et al. (2012) Decreased Serum Total T3 Level in Hepatitis B and C Related Cirrhosis by Severity of Liver Damage. Annals of Hepatology, 11, 667-671.

[10] Eshraghian, A. and Taghavi, S.A. (2014) Systematic Review: Endocrine Abnormalities in Patients with Liver Cirrhosis. Archives of Iranian Medicine, 17, 713-721.

[11] Antonelli, A., et al. (2004) Thyroid Involvement in Patients with Overt HCV-Related Mixed Cryoglobulinaemia. QJM, 97, 499-506. http://dx.doi.org/10.1093/qjmed/hch088

[12] Kabadi, U.M. and Premachandra, B.N. (1983) Serum T3 and Reverse T3 Levels in Hepatic Cirrhosis: Relation to Hepatocellular Damage and Normalization on Improvement in Liver Dysfunction. The American Journal of Gastroenterology, 78, 750-755.

[13] Moustafa, A.H., Ali, E.M., Mohamed, T.M. and Abdou, H.I. (2009) Oxidative Stress and Thyroid Hormones in Patients with Liver Diseases. European Journal of Internal Medicine, 20, 703-708. http://dx.doi.org/10.1016/j.ejim.2009.08.003

[14] El-Kabbany, Z.A., et al. (2012) Thyroid and Hepatic Haemodynamic Alterations among Egyptian Children with Liver Cirrhosis. ISRN Gastroenterology, 2012, 595-734.

[15] Guven, K., Kelestimur, F. and Yucesoy, M. (1993) Thyroid Function Tests in Non-Alcoholic Cirrhotic Patients with Hepatic Encephalopathy. European Journal of Internal Medicine, 2, 83-85.

[16] Borzio, M., Caldara, R., Borzio, F., Piepoli, V., Rampini, P. and Ferrari, C. (1983) Thyroid Function Tests in Chronic Liver Disease: Evidence for Multiple Abnormalities despite Clinical Euthyroidism. Gut, 24, 631-636. http://dx.doi.org/10.1136/gut.24.7.631

[17] Yang, R., Shan, Z.Y., Li, Y.S., Fan, C.L., Li, C.Y. and Teng, W.P. (2011) Prevalence of Thyroid Autoantibodies in Hepatitis $\mathrm{C}$ and Hepatitis B Infection in China. Internal Medicine, 50, 811-815. http://dx.doi.org/10.2169/internalmedicine.50.4870

[18] Ilias, I., et al. (2007) Contribution of Endocrine Parameters in Predicting Outcome of Multiple Trauma Patients in an Intensive Care Unit. Hormones, 6, 218-226.

[19] Huang, M.J. and Liaw, Y.F. (1995) Clinical Associations between Thyroid and Liver Diseases. Journal of Gastroenterology and Hepatology, 10, 344-350. http://dx.doi.org/10.1111/j.1440-1746.1995.tb01106.x 